\title{
A Simple Tool for Qualitatively Testing, Quantitatively Measuring, and Normatively Justifying Savage's Subjective Expected Utility
}

\author{
VERONIKA KÖBBERLING \\ PETER P. WAKKER* \\ P.P.Wakker@uva.nl (www.fee.uva.nl/creed/wakker) \\ Department of Quantitative Economics, University of Maastricht, P.O. Box 616, Maastricht 6200 MD, \\ The Netherlands
}

\begin{abstract}
This paper introduces a new preference condition that can be used to justify (or criticize) expected utility. The approach taken in this paper is an alternative to Savage's, and is accessible to readers without a mathematical background. It is based on a method for deriving "comparisons of tradeoffs" from ordinal preferences. Our condition simplifies previously-published tradeoff conditions, and at the same time provides more general and more powerful tools to specialists. The condition is more closely related to empirical methods for measuring utility than its predecessors. It provides a unifying tool for qualitatively testing, quantitatively measuring, and normatively justifying expected utility.
\end{abstract}

Keywords: cardinal utility, expected utility, tradeoff consistency, strength of preference

JEL Classification: $\quad$ D81, C60

A central topic of debate in decision theory is the normative status of expected utility. Expected utility provides a general model for decisions under uncertainty and was considered normative by its early contributors (Ramsey, 1931; de Finetti, 1937; von Neumann and Morgenstern, 1944; Savage, 1954; Raiffa, 1968). Many have, however, challenged the normative status of expected utility (Allais, 1953; Ellsberg, 1961; Epstein, 1992; Loomes and Sugden, 1982; Machina, 1982; Schmeidler, 1989). The debate has usually centered around the plausibility of the preference conditions that characterize the model. Such conditions show how the model can be justified or criticized in terms of directly observable preferences.

Von Neumann and Morgenstern (1944) provided a simple and appealing characterization of expected utility by means of their independence preference condition. However, their characterization only applies to decision under risk, i.e. the special case of known probabilities. Economically more relevant is the case of uncertainty, where probabilities are unknown. Characterizations and measurements for this case are more complex. Savage (1954) provided a first characterization of expected utility for uncertainty. We 
propose an alternative characterization, based on a method for measuring utility under uncertainty. This method uses comparisons of "tradeoffs" (Wakker, 1984), by means of which orderings of utility differences can be inferred from ordinal preferences between gambles. Wakker (1989) showed that expected utility holds if and only if such comparisons of tradeoffs do not lead to contradictions. A variation of this method, renamed tradeoff consistency, was used by Tversky and Kahneman (1992) to characterize the new version of prospect theory. We simplify and generalize the tradeoff conditions published before.

This paper concentrates on the intuition of the tradeoff condition, leading to a new intuitive foundation for expected utility. In short, Savage's (1954) event-independent ordering of outcomes (P3) is extended to an event-independent ordering of strengths of preferences between outcomes. We emphasize that these strengths of preferences are derived only from ordinal empirical inputs, and are not assumed as empirical primitives. Our approach, therefore, remains within the revealed preference paradigm. A consistency requirement for the strength of preference ordering implies both the existence of utility and the existence of subjective probabilities. It thus generates the whole subjective expected utility model of Savage (1954). Mathematical generalizations will be given in the follow-up paper Köbberling and Wakker (2003).

While our approach is simpler than earlier approaches and, therefore, more easily accessible to nonspecialists, it is at the same time more general than earlier approaches in a mathematical sense. That is, it derives the same implications from weaker assumptions, thereby providing more powerful techniques for specialists. The obtained simplification allows for a new characterizing condition, alternative to tradeoff consistency: Expected utility holds if and only if the revealed "equivalences of tradeoffs" satisfy transitivity.

A further advantage of our tradeoff consistency is that it is closer to empirical measurements of utility than its predecessors, e.g. those in Wakker $(1984,1989,1994)$, Tversky and Kahneman (1992) and Abdellaoui (2002). This paper can be considered the theoretical counterpart to the empirical utility measurement of Wakker and Deneffe (1996).

We hope that the tradeoff technique can appeal to a basic concept of cardinal utility, prior to risk or uncertainty, and can thus formalize an intuition described by Broome (1991, p. 147). A similar concept of utility was advocated by Harsanyi (1978) for expected utility, and by Allais (1953) and Loomes and Sugden (1982) in their derivations of nonexpected utility models. The tradeoff technique may provide a link between risky ${ }^{1}$ utility, which it formally reveals, and other concepts of cardinal utility (discussed by Fishburn, 1989; Ng, 1997; Samuelson, 1937, p. 161; Zeuthen, 1937), while remaining within the revealed-preference paradigm. In a follow-up paper, Abdellaoui, Barrios, and Wakker (2003) tested this claim experimentally. They found that risky utilities, measured by means of the techniques of this paper, did indeed agree well with riskless utilities measured through direct strength of preference questions. The latter were not based on revealed preferences but on direct judgments.

Like earlier derivations based on tradeoff consistency, our derivation can deal with finite state spaces, extending Savage (1954) who required an infinite state space. Our derivation can also deal with infinite state spaces, using Wakker's (1993, Corollary 2.14) technique. Thus, a modification of Savage (1954) results that allows for atoms. The above result can 
also be interpreted as a modification of Anscombe and Aumann's (1963) characterization of subjective expected utility that avoids their two-stage setup and their probability-mixing of outcomes. A third interpretation is that the above theorem extends de Finetti's (1937) book-making argument, when reformulated as a coherence condition for individual choice, to the case of nonlinear utility. This was the original motivation for the second author of this paper to initiate the tradeoff approach. Other derivations of subjective expected utility that can be interpreted in the same ways are in Grodal (1978), Nakamura (1990), Gul (1992) and Chew and Karni (1994).

The tradeoff method can be used to empirically classify deviations from expected utility. For example, if tradeoff consistency can be violated in general but is never violated when the considered gambles are "comonotonic" (defined in Schmeidler, 1989), then a rank-dependent model can be shown to apply (Köbberling and Wakker, 2003). If tradeoff consistency can be violated when the considered gambles are comonotonic but only if they are not co-signed (co-signed means that there is no state yielding a gain under one gamble and a loss under another), then prospect theory holds (Köbberling and Wakker, 2003). Under betweenness theories (Dekel, 1986; Epstein, 1992), tradeoff consistency is never violated within indifference classes (Gilboa, Schmeidler, and Wakker, 2002; Lemma A.5), but can be between.

\section{The intuition underlying our condition}

Measurements of strength of preferences have been controversial in the economic literature. For example, assume that an agent judges that his strength of preference for $\$ 100$ instead of $\$ 0$ exceeds that for $\$ 1101$ instead of $\$ 1000$. Such subjective judgments cannot be revealed from observed choice. While they may be psychologically meaningful (Pareto, 1906, p. 191 in 1971 translation), it became generally accepted that they should not be part of the economic domain (Hicks and Allen, 1934).

This section proposes a different concept of strength of preference, one that can be revealed from preferences under uncertainty. For simplicity of presentation, only monetary outcomes are considered henceforth.

Assume that a decision maker has to choose between state-contingent assets called gambles. For instance, a gamble may be $(A, 310 ; B, 0)$, yielding $\$ 310$ if event $A$ obtains (e.g., candidate 1 will win the elections) and $\$ 0$ if event $B$ obtains (another candidate will win). Subjective ${ }^{2}$ expected utility requires that a probability $P$ is assigned to each event and a utility $U$ to each outcome. If no probabilities of events are known, subjective probabilities have to be assigned that best reflect the degree of belief of the decision maker. Gambles are evaluated by their probability-weighted average utility, e.g. $P(A) U(310)+P(B) U(0)$ for the gamble just described. From available gambles, the one with the highest expected utility is chosen.

In the following analysis, we do not assume expected utility but instead consider preference conditions that are argued to be reasonable for normative purposes. We then show that they imply expected utility. The intuitions underlying our analysis are similar to those in Loomes and Sugden (1982), Broome (1991, p. 146/147) and Baron (1994, Section 17.1.5). To explain the basic tool of our method, we consider the following two indifferences, 


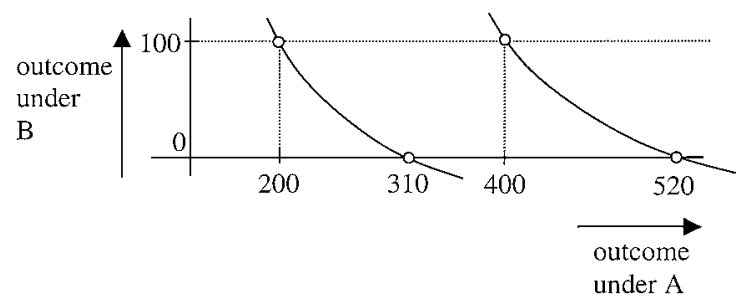

Figure 1. The indifference curves reveal $[310 ; 200] \sim^{*}[520 ; 400]$.

depicted in Figure 1.

$$
\begin{aligned}
& (A, 310 ; B, 0) \sim(A, 200 ; B, 100) \quad \text { and } \\
& (A, 520 ; B, 0) \sim(A, 400 ; B, 100) .
\end{aligned}
$$

In both indifferences, event $B$ provides an argument against the left gamble because then outcome 0 instead of 100 is received. Henceforth $[0 ; 100]$ is a linguistic abbreviation of "receiving outcome 0 instead of outcome 100." In both indifferences, event $A$ provides an argument in favor of the left gamble corresponding to [310;200] (receiving outcome 310 instead of outcome 200) in the first indifference and to [520;400] in the second.

Obviously, the strength of the argument against the left gamble provided by $[0 ; 100]$ depends not only on how much worse it is to receive 0 instead of 100 but also on event $B$, in particular its perceived likelihood. (The likelihood of) $A$ is similarly relevant to the weight of the arguments provided by [310;200] and [520;400], respectively. At any rate, the first indifference suggests the following interpretation:

([310;200] contingent on event $A)$ exactly offsets ([0;100] contingent on event $B$ ).

Similar "statewise" comparisons are described by Tversky (1969, p. 41) and are central in regret theory (Loomes and Sugden, 1982) and in Fishburn's skew-symmetric bilinear theory (Fishburn and LaValle, 1988). The second indifference can be interpreted likewise, i.e.

([520;400] contingent on event $A$ ) exactly offsets ([0;100] contingent on event $B$ ).

These two interpretations together suggest that $[520 ; 400]$ can do exactly the same as $[310 ; 200]$, i.e. offset $[0 ; 100]$ contingent on event $B$ while they $([520 ; 400]$ and $[310 ; 200])$ are contingent on the same event $A$. We interpret this as $[310 ; 200]$ being an equally good improvement as $[520 ; 400]$, and use the notation

$$
[310 ; 200] \sim^{*}[520 ; 400]
$$


as a linguistic abbreviation of this interpretation. Note how $[0 ; 100]$ contingent on event $B$ served as a gauge to establish Eq. (5).

We next formalize the concepts discussed so far. The relationships $\sim *$ can be derived from other gambles than the ones just considered. Consider general multiple-outcome gambles of the form $\left(E_{1}, \alpha ; E_{2}, x_{2} ; \ldots ; E_{n}, x_{n}\right)$, where $E_{1}, \ldots, E_{n}$ is a list of exhaustive and mutually exclusive events. The gamble yields $\$ \alpha$ if $E_{1}$ obtains, $\$ x_{2}$ if $E_{2}$ obtains, ..., and $\$ x_{n}$ if $E_{n}$ obtains. Consider the following indifferences.

$$
\begin{aligned}
& \left(E_{1}, \alpha ; E_{2}, x_{2} ; \ldots ; E_{n}, x_{n}\right) \sim\left(E_{1}, \beta ; E_{2}, y_{2} ; \ldots ; E_{n}, y_{n}\right) \text { and } \\
& \left(E_{1}, \gamma ; E_{2}, x_{2} ; \ldots ; E_{n}, x_{n}\right) \sim\left(E_{1}, \delta ; E_{2}, y_{2} ; \ldots ; E_{n}, y_{n}\right) .
\end{aligned}
$$

We formally write

$$
[\alpha ; \beta] \sim^{*}[\gamma ; \delta]
$$

whenever we can find events $E_{1}, \ldots, E_{n}$ and outcomes $x_{2}, \ldots, x_{n}, y_{2}, \ldots, y_{n}$ such that the above indifferences hold. The interpretation is the same as discussed at the beginning of this section. The two indifferences in Eqs. (1) and (2) are a special case of those in Eqs. (6) and (7) (take $n=2, E_{1}=A, E_{2}=B, \alpha=310, \beta=200, \gamma=520, \delta=400, x_{2}=0, y_{2}=100$ ). In Eqs. (6) and (7), $[\alpha ; \beta]$ and $[\gamma ; \delta]$, contingent on event $E_{1}$, offset the same gauge (receiving the $x$ outcomes instead of the $y$ outcomes) contingent on not- $E_{1}$. The latter gauge is more complex than what we saw before, with various outcomes and events involved, but this does not change the reasoning. The following observation may further clarify the meaning of the $\sim^{*}$ relation. The algebraic demonstration is given in the main text because it matches the psychological interpretation of the above operations.

Observation 1. Under expected utility $[\alpha ; \beta] \sim^{*}[\gamma ; \delta]$ implies $U(\alpha)-U(\beta)=U(\gamma)-$ $U(\delta)$.

Demonstration. The indifference in Eq. (1) implies

$$
P(A)(U(310)-U(200))=P(B)(U(100)-U(0))
$$

(compare the interpretation in Eq. (3)). The indifference in Eq. (2) implies

$$
P(A)(U(520)-U(400))=P(B)(U(100)-U(0))
$$

(compare the interpretation in Eq. (4)). Dropping a common factor $P(A)$, we obtain the following quantitative counterpart to the indifference $\sim^{*}$ in Eq. (5).

$$
U(310)-U(200)=U(520)-U(400)
$$

Similar algebraic manipulations can be applied to Eqs. (6) and (7). 
If the interpretation of the relation $\sim^{*}$ is accepted, then transitivity of this relation is a reasonable requirement for normative purposes. That is, if $[\alpha ; \beta]$ can offset the same gauge as $[\gamma ; \delta]$ in one situation, and $[\gamma ; \delta]$ can offset another "same gauge" as $[\sigma ; \tau]$ in another situation, then it seems reasonable that $[\alpha ; \beta]$ can offset the same gauges as $[\sigma ; \tau]$.

In the same vein as above, the relationships $\sim^{*}$ should not reveal contradictory orderings in the following sense. Assume that we have the two indifferences in Eqs. (6) and (7) with $\alpha=315, \beta=200, \gamma=520$, and $\delta=400$, so that [315;200] $\sim^{*}$ [520;400] follows. Assume that we also have the two indifferences at the beginning of this section, implying $[310 ; 200] \sim^{*}[520 ; 400]$. In a way similar to transitivity, this generates the undesirable suggestion that $[315 ; 200]$ would be an equally good improvement as [310;200], both being equally good as $[520 ; 400]$. To exclude such undesirable suggestions, we define tradeoff consistency as the requirement that $\left[\alpha^{\prime} ; \beta\right] \sim^{*}[\gamma ; \delta]$ and $[\alpha ; \beta] \sim^{*}[\gamma ; \delta]$ does not hold for any $\alpha^{\prime}>\alpha .{ }^{3}$ Because of symmetries, tradeoff consistency implies that worsening outcome $\alpha$ also breaks the relationship $\alpha \beta \sim^{*} \gamma \delta$, and that similar conditions hold for $\beta, \gamma, \delta$ as for $\alpha$. In words:

Definition 2. Tradeoff consistency holds if: improving an outcome in a $\sim^{*}$ relationship breaks the relationship.

As it turns out, transitivity and tradeoff consistency of $\sim^{*}$ need not hold for general preferences. We will see that each of these two conditions holds in isolation, under some natural conditions, if and only if expected utility holds. In particular, the two conditions are equivalent. The aforementioned natural conditions are weak ordering (completeness of preference for every pair of gambles and transitivity), monotonicity (the more money the better), and continuity (small changes in outcomes do not generate large changes in preference).

Theorem 3. Assume that preferences satisfy weak ordering, monotonicity, and continuity. The following three statements are equivalent.

(i) Subjective expected utility holds.

(ii) Tradeoff consistency holds.

(iii) $\sim^{*}$ is transitive.

If tradeoff consistency or transitivity of the revealed orderings of tradeoffs is deemed (un)desirable in an empirical or normative sense, then so should expected utility be. We sketch the proof of the theorem in the main text because it illustrates how to measure probabilities and utilities empirically. Topological and other technical aspects of the proof are elaborated in Köbberling and Wakker (2003), where also nonmonetary outcomes are considered.

To define utility $U$, we set $U\left(x^{0}\right)=0$ for some $x^{0}$, and $U\left(x^{1}\right)=1$ for some $x^{1}>x^{0}$. For all integers $j$ (also negative), we take $x^{j}$ such that $x^{j} x^{j-1} \sim^{*} x^{1} x^{0}$, and define $U\left(x^{j}\right)=j$. If utility is bounded there will be bounds on the indexes $j$ for which such $x^{j}$ can be defined. Next, we refine the domain of $U$, starting with outcomes $y^{j}$ s where $U$ is to take the values $j / 2$. First define $y^{1}$ such that $x^{1} y^{1} \sim^{*} y^{1} x^{0}$, which will imply that $U\left(y^{1}\right)=1 / 2$ so that $y^{1}$ can be interpreted as a preference-midpoint between $x^{0}$ and $x^{1}$. Then define $y^{0}=x^{0}$, and a sequence $y^{j}$ that satisfies $y^{j} y^{j-1} \sim^{*} y^{1} x^{0}$ for all $j$. Define $U\left(y^{j}\right)=j / 2$ for all 
$j$. With further similar refinements, $U$ can be defined to any degree of specificity. By limiting procedures and continuity, $U$ is extended to all outcomes. Tradeoff consistency, or transitivity of $\sim^{*}$, implies, together with the other conditions, that the construction of utility does not run into contradictions. For instance, it can be demonstrated that $y^{2 k}=x^{k}$ not only for $k=1$, but also for all other $k$. The subjective probability of each event $A$ is the utility gained for sure that is equivalent to gaining one utility unit conditional on event $A$. Again, tradeoff consistency together with the other conditions ensures that this definition of subjective probabilities does not run into contradictions and yields an additive probability measure. Finally, tradeoff consistency also ensures that the expectation generated by the constructed probabilities and utilities properly evaluates gambles.

\section{Discussion}

Jonathan Baron (personal communication) pointed out an interesting analogy between tradeoff consistency and Savage's (1954) P3, a well-known monotonicity condition. Taking an example with nonmonetary outcomes, from $\left(A\right.$, wine; $A^{c}$, water $) \succeq\left(A\right.$, beer; $A^{c}$, water $)$ we can derive the following preference ordering over outcomes: wine $\succeq$ beer. Savage's P3 requires that such derived preferences over outcomes be independent of (the non-null) event $A$ and of the common outcomes (water in our case) outside of $A$. Tradeoff consistency extends this independence to derived orderings of strengths of preferences. The resulting condition is, of course, considerably more restrictive, but the intuitive analogy is interesting. Recently, Bouyssou and Pirlot (2003) formalized this intuition. Rewrite, for fixed $\left(E_{1}, \ldots, E_{n}\right)$, an indifference $\left(E_{1}, \alpha ; \ldots ; E_{n}, x_{n}\right) \sim\left(E_{1}, \beta ; \ldots ; E_{n}, y_{n}\right)$ as $\left(\alpha, \beta ; \ldots ; x_{n}, y_{n}\right) \in \sim$. They showed that tradeoff consistency amounts to a weak separability condition for the pair $(\alpha, \beta)$ in this indifference.

For completeness, let us briefly explain how the conditions of this paper are related to earlier versions of tradeoff consistency, used by Wakker (1989) and others. Those conditions used two $*$-preference orderings over strengths of preferences instead of our $\sim^{*}$, a strict one with weak preference $\succeq$ in Eq. (6) and strict preference $\prec$ in Eq. (7), and a weak one with weak preferences $\succeq$ and $\preceq$ in those equations. Wakker (1989, Theorem IV.2.7) used absence of contradictions between these relationships to characterize subjective expected utility. Theoretical follow-up papers used similar preference conditions (Abdellaoui, 2002; Bleichrodt and Quiggin, 1999; Bleichrodt and Miyamoto, 2003; Ebert, 2002; Gilboa, Schmeidler, and Wakker, 2002; Karni, 2003; Prelec, 1998; Schmidt, 2003; Schmidt and Zank, 2001; Tversky and Kahneman, 1992; Wakker and Tversky, 1993). The version of this paper is less restrictive and more powerful, closer to empirical applications, and simpler because it avoids asymmetries in the definition. Skiadas (1997) used conditions closer to those of this paper, also in terms of indifferences, in combination with other axioms.

We next discuss a number of empirical studies that used "tradeoff" reasonings to measure utility. As we saw, $\sim^{*}$ reveals identities of utility differences under expected utility. Wakker and Deneffe (1996) elicited values $x^{0}, \ldots, x^{4}$ as described in the proof of Theorem 3, at the end of Section 1. Normalizing utility such that $U\left(x^{0}\right)=0$ and $U\left(x^{4}\right)=1$ implies $U\left(x^{j}\right)=j / 4$ for all $j$. We think that measurements by means of the relation $\sim^{*}$ encourage subjects to adopt rational procedures of weighing positive against negative arguments, rather 
than resorting to irrational noncompensatory heuristics (compare Broome, 1991, p. 7). In this manner, the relation $\sim *$ can be suited for constructive elicitations of preferences (Payne, Bettman, and Schkade, 1999).

Fennema and van Assen (1998) used the tradeoff method to investigate whether utility for losses is convex or concave. Whereas economic studies have usually assumed concave utility, psychologists found evidence of convex utilities for losses (Tversky and Kahneman, 1992). The traditional methods for measuring utility have been confounded by other aspects of risk attitude such as probability transformations. Fennema and van Assen's study avoided a number of such confounding aspects and found more convexity than concavity for utility in the loss domain. Other works that used the tradeoff method for measuring utility include Fennema and van Assen (1998), Abdellaoui (2000), Abdellaoui, Vossmann, and Weber (2003), Bleichrodt and Pinto (2000), de Blaeij and van Vuuren (2003), Etchart (2003) and Van Assen and Snijders (2001). Abdellaoui and Munier (1999) used the method in a dual manner to measure probability transformations.

Tradeoff consistency can be criticized for being based on the relation $\sim^{*}$ that is not a primitive but is derived from the preference relation. The derivation is, however, simple, and substitution of the definition of $\sim^{*}$ in tradeoff consistency directly yields a tractable restatement of tradeoff consistency in terms of preferences: Eqs. (6) and (7) and

$$
\left(A_{1}, \alpha ; A_{2}, v_{2} ; \ldots ; A_{m}, v_{m}\right) \sim\left(A_{1}, \beta ; A_{2}, w_{2} ; \ldots ; A_{m}, w_{m}\right)
$$

should always imply

$$
\left(A_{1}, \gamma ; A_{2}, v_{2} ; \ldots ; A_{m}, v_{m}\right) \sim\left(A_{1}, \delta ; A_{2}, w_{2} ; \ldots ; A_{m}, w_{m}\right)
$$

Tradeoff consistency is more complex that the independence condition of von Neumann and Morgenstern (1944). In return, however, it solves a more complex problem. In von Neumann and Morgenstern's model, probabilities are given, so that characterizing expected utility amounts to solving linear inequalities. For tradeoff consistency, no probabilities are given, and the condition serves to solve nonlinear inequalities. It delivers not only utilities as does von Neumann and Morgenstern's independence, but it also delivers subjective probabilities when these were not given beforehand.

Empirical tests of tradeoff consistency are tractable, and naturally result from crosschecking in quantitative utility measurements. Such tests were conducted as follows in an informal experiment, where a pair of outcomes $(x, y)$ denotes a fifty-fifty gamble yielding $x$ or $y$. First, outcomes $10,000=x^{0}, \ldots, x^{4}$ were elicited such that $\left(1000, x^{j}\right) \sim\left(8000, x^{j-1}\right)$ for $j=1, \ldots, 4$, implying $\left[x^{j} ; x^{j-1}\right] \sim^{*}\left[x^{1} ; x^{0}\right]$ for all $j$. Second, after some intermediate questions to reduce memory effects, the experimentor chose a $g>x^{4}$ and $G, y^{2}, \ldots, y^{4}$ were elicited such that $\left(g, y^{j}\right) \sim\left(G, y^{j-1}\right)$ for $j=1$ to 4 , where $y^{0}=x^{0}$ and $y^{1}=x^{1}$ was taken. Tradeoff consistency requires that $y^{j}=x^{j}$ also for $j=2, \ldots, 4$. The null hypothesis of these equalities was not rejected. The discrepancies between the $y^{j} \mathrm{~s}$ and the $x^{j} \mathrm{~S}$ were smaller than the discrepancies caused by some framing effects that were also tested and that were significant. Such findings, if confirmed by thorough experiments, suggest that deviations from expected utility primarily concern basic rationality conditions such as 
transitivity and framing invariance, and to a lesser extent concern sophisticated conditions such as tradeoff consistency, the sure-thing principle, etc.

\section{Conclusion}

It will not take much time to explain to students the notation in Eqs. (6) and (7), the intuition of Eq. (8), and the verbal statement of Definition 2. In this manner, Savage's (1954) derivation of both utilities and subjective probabilities, and of the expected utility criterion, can be explained in a short time span (LeRoy and Werner, 2000, Section 8.6).

We hope that the $\sim^{*}$ relation, while formally revealing risky utility differences, nevertheless appeals to an intuition of strength of preference and thereby can help to relate risky and riskless utility. In general, we hope that one concept of cardinal utility, relevant for risky decisions, intertemporal decisions, welfare evaluations, and central in all social sciences, will emerge from current decision theories, an index of goodness in the sense of Bentham (1789), Broome (1991) and Robson (2001).

\section{Acknowlegdment}

Jonathan Baron and Han Bleichrodt made helpful comments.

\section{Notes}

1. It is customary to let "risky utility" refer to both utility for risk and utility for uncertainty, and let "riskless utility" refer to the large and diverse class of cardinal utilities that may arise under conditions without risk, such as intertemporal or interpersonal aggregations. Although the terminology may be misleading for the purposes of this paper, which concerns general uncertainty, it is so customary that we will adhere to it.

2. Henceforth, we usually drop "subjective."

3. $\alpha^{\prime} \succ \alpha$ for general nonmonetary outcomes.

\section{References}

Abdellaoui, Mohammed. (2000). "Parameter-Free Elicitation of Utilities and Probability Weighting Functions," Management Science 46, 1497-1512.

Abdellaoui, Mohammed. (2002). "A Genuine Rank-Dependent Generalization of the von Neumann-Morgenstern Expected Utility Theorem," Econometrica 70, 717-736.

Abdellaoui, Mohammed, Carolina Barrios, and Peter P. Wakker. (2003). "Reconciling Introspective Utility With Revealed Preference: Experimental Arguments Based On Prospect Theory,” GRID, Cachan, France.

Abdellaoui, Mohammed and Bertrand R. Munier. (1999). "How Consistent Are Probability Tradeoffs in Individual Preferences under Risk? Some Preliminary Results.” In Mark J. Machina and Bertrand R. Munier (eds.), Beliefs, Interactions and Preferences in Decision-Making, Dordrecht, The Netherlands: Kluwer Academic Publishers.

Abdellaoui, Mohammed, Frank Vossmann, and Martin Weber. (2003). "Choice-Based Elicitation and Decomposition of Decision Weights for Gains and Losses under Uncertainty," GRID, Cachan, France.

Allais, Maurice. (1953). "Fondements d'une Théorie Positive des Choix Comportant un Risque et Critique des Postulats et Axiomes de l'Ecole Américaine," Colloques Internationaux du Centre National de la Recherche Scientifique Econométrie, 40, 257-332. Paris: Centre National de la Recherche Scientifique. Translated into English, with additions, as "The Foundations of a Positive Theory of Choice Involving Risk and a Criticism of 
the Postulates and Axioms of the American School.' In Maurice Allais and Ole Hagen (eds.) (1979). Expected Utility Hypotheses and the Allais Paradox, Dordrecht, The Netherlands: Reidel, pp. 27-145.

Anscombe, F.J. and Robert J. Aumann. (1963). “A Definition of Subjective Probability,” Annals of Mathematical Statistics 34, 199-205.

Baron, Jonathan. (1994). Thinking and Deciding, 2nd edn. Cambridge: Cambridge University Press.

Bentham, Jeremy. (1789). The Principles of Morals and Legislation. At the Clarendon Press, Oxford.

Bleichrodt, Han and José Luis Pinto. (2000). "A Parameter-Free Elicitation of the Probability Weighting Function in Medical Decision Making," Management Science 46, 1485-1496.

Bleichrodt, Han and John Quiggin. (1999). "Life-Cycle Preferences over Consumption and Health: When is Cost-Effectiveness Analysis Equivalent to Cost-Benefit Analysis?” Journal of Health Economics 18, 681-708.

Bleichrodt, Han and John Miyamoto. (2003). "A Characterization of Quality-Adjusted Life-Years under Cumulative Prospect Theory," Mathematics of Operations Research 28, 181-193.

Broome, John R. (1991). Weighing Goods. Oxford: Basil Blackwell.

Bouyssou, Denis and Marc Pirlot. (2003). “A Note on Wakker's Cardinal Coordinate Independence,” LAMSADE, Université Paris Dauphine, Paris, France.

Chew, Soo Hong and Edi Karni. (1994). "Choquet Expected Utility with a Finite State Space: Commutativity and Act-Independence,” Journal of Economic Theory 62, 469-479.

de Blaeij, Arianne T. and Daniel J. van Vuuren. (2003). "Risk Perception of Traffic Participants," Accident Analysis and Prevention 35, 167-175.

de Finetti, Bruno. (1937). "La Prévision: Ses Lois Logiques, ses Sources Subjectives," Annales de l'Institut Henri Poincaré 7, 1-68. Translated into English by Henry E. Kyburg, "Foresight: Its Logical Laws, its Subjective Sources.” In Henry E. Kyburg and Howard E. Smokler (eds.) (1964). Studies in Subjective Probability, New York: Wiley, pp. 53-118; 2nd edn. 1980, Krieger, New York.

Dekel, Eddie. (1986). "An Axiomatic Characterization of Preferences Under Uncertainty: Weakening the Independence Axiom," Journal of Economic Theory 40, 304-318.

Ebert, Udo. (2002). "Social Welfare, Inequality, and Poverty when Needs Differ: Rank-Dependent Evaluation Functions," Social Choice and Welfare (forthcoming).

Ellsberg, Daniel. (1961). "Risk, Ambiguity and the Savage Axioms," Quarterly Journal of Economics 75, 643-669.

Epstein, Larry G. (1992). "Behavior Under Risk: Recent Developments in Theory and Applications." In JeanJacques Laffont (ed.), Advances in Economic Theory. Cambridge, UK: Cambridge University Press, pp. 1-63.

Etchart, Nathalie. (2002). "Is Probability Weighting Sensitive to the Magnitude of Consequences? An Experimental Investigation on Losses," Journal of Risk and Uncertainty (forthcoming).

Fennema, Hein and Marcel A.L.M. van Assen. (1998). "Measuring the Utility of Losses by Means of the Tradeoff Method," Journal of Risk and Uncertainty 17, 277-295.

Fishburn, Peter C. (1989). "Retrospective on the Utility Theory of von Neumann and Morgenstern," Journal of Risk and Uncertainty 2, 127-158.

Fishburn, Peter C. and Irving H. LaValle. (1988). "Context-Dependent Choice with Nonlinear and Nontransitive Preferences," Econometrica 56, 1221-1239.

Gilboa, Itzhak, David Schmeidler, and Peter P. Wakker. (2002). "Utility in Case-Based Decision Theory," Journal of Economic Theory 105, 483-502.

Grodal, Birgit. (1978). “Some Further Results on Integral Representation of Utility Functions,” Institute of Economics, University of Copenhagen, Copenhagen.

Gul, Faruk. (1992). "Savage's Theorem with a Finite Number of States," Journal of Economic Theory 57, 99-110. ("Erratum," Journal of Economic Theory 61, 1993, 184.)

Harsanyi, John C. (1978). "Bayesian Decision Theory and Utilitarian Ethics," American Economic Review, Papers and Proceedings 68, 223-228.

Hicks, John R. and Roy G.D. Allen. (1934). "A Reconsideration of the Theory of Value: I; II," Economica 1, 52-75, 196-219.

Karni, Edi. (2003). “On the Representation of Beliefs by Probabilities,” Journal of Risk and Uncertainty 26, 17-38. Köbberling, Veronika and Peter P. Wakker. (2003). "Preference Foundations for Nonexpected Utility: A Generalized and Simplified Technique," Mathematics of Operations Research 28, 395-423.

LeRoy, Stephen F. and Jan Werner. (2000). Principles of Financial Economics. New York: Cambridge University Press. 
Loomes, Graham and Robert Sugden. (1982). "Regret Theory: An Alternative Theory of Rational Choice Under Uncertainty," Economic Journal 92, 805-824.

Machina, Mark J. (1982). “Expected Utility’ Analysis without the Independence Axiom,” Econometrica 50, 277-323.

Nakamura, Yutaka. (1990). "Subjective Expected Utility with Non-Additive Probabilities on Finite State Spaces," Journal of Economic Theory 51, 346-366.

Ng, Yew-Kwang. (1997). "A Case for Happiness, Cardinalism, and Interpersonal Comparability," Economic Journal 107, 1848-1858.

Pareto, Vilfredo. (1906). Manuele di Economia Politica. Piccolo Biblioteca Scientifica, Milan. Translated into English by Ann S. Schwier (1971). Manuel of Political Economy. London: MacMillan.

Payne, John W., James R. Bettman, and David A. Schkade. (1999). "Measuring Constructed Preferences: Towards a Building Code," Journal of Risk and Uncertainty 19, 243-270.

Prelec, Drazen. (1998). “The Probability Weighting Function,” Econometrica 66, 497-527.

Raiffa, Howard. (1968). Decision Analysis. London: Addison-Wesley.

Ramsey, Frank P. (1931). “Truth and Probability.” In The Foundations of Mathematics and other Logical Essays. London: Routledge and Kegan Paul, pp. 156-198. Reprinted in Henry E. Kyburg and Howard E. Smokler (eds.) (1964). Studies in Subjective Probability. New York: Wiley, pp. 61-92.

Robson, Arthur J. (2001). "Why Would Nature Give Individuals Utility Functions?" The Journal of Political Economy 109, 900-914.

Samuelson, Paul A. (1937). "A Note on Measurement of Utility," Review of Economic Studies 4(2), $155-161$.

Savage, Leonard J. (1954). The Foundations of Statistics. New York: Wiley (2nd edn., 1972, New York: Dover).

Schmeidler, David. (1989). "Subjective Probability and Expected Utility without Additivity," Econometrica 57, 571-587.

Schmidt, Ulrich. (2003). "Reference-Dependence in Cumulative Prospect Theory," Journal of Mathematical Psychology 47, 122-131.

Schmidt, Ulrich and Horst Zank. (2001). "A New Axiomatization of Rank-Dependent Expected Utility with Tradeoff Consistency for Equally Likely Outcomes," Journal of Mathematical Economics 35, 483-491.

Skiadas, Costis. (1997). "Subjective Probability Under Additive Aggregation of Conditional Preferences," Journal of Economic Theory 76, 242-271.

Tversky, Amos. (1969). "Intransitivity of Preferences," Psychological Review 76, 31-48.

Tversky, Amos and Daniel Kahneman. (1992). "Advances in Prospect Theory: Cumulative Representation of Uncertainty," Journal of Risk and Uncertainty 5, 297-323.

Van Assen, Marcel A.L.M., and Chris Snijders. (2003). "Effects of Risk Preferences in Repeated Social Dilemmas: A Game-Theoretic Analysis and Evidence from Two Experiments." In Ramzi Suleiman, David V. Budescu, and David Messick (eds.), Contemporary Psychological Research on Social Dilemmas. Dordrecht: Kluwer (forthcoming).

von Neumann, John and Oskar Morgenstern. (1944, 1947, 1953). Theory of Games and Economic Behavior. Princeton NJ: Princeton University Press.

Wakker, Peter P. (1984). "Cardinal Coordinate Independence for Expected Utility," Journal of Mathematical Psychology 28, 110-117.

Wakker, Peter P. (1989). Additive Representations of Preferences, A New Foundation of Decision Analysis. Dordrecht: Kluwer Academic Publishers.

Wakker, Peter P. (1993). "Unbounded Utility for Savage's "Foundations of Statistics," and other Models," Mathematics of Operations Research 18, 446-485.

Wakker, Peter P. (1994). "Separating Marginal Utility and Probabilistic Risk Aversion," Theory and Decision 36, $1-44$.

Wakker, Peter P. and Daniel Deneffe. (1996). "Eliciting von Neumann-Morgenstern Utilities when Probabilities Are Distorted or Unknown," Management Science 42, 1131-1150.

Wakker, Peter P. and Amos Tversky. (1993). “An Axiomatization of Cumulative Prospect Theory,” Journal of Risk and Uncertainty 7, 147-176.

Zeuthen, Frederik. (1937). "On the Determinateness of the Utility Function,” Review of Economic Studies 4, 236-239. 